\title{
Adolescent Girls Empowerment Program (AGEP): Endline results-Executive summary
}

Population Council

Follow this and additional works at: https://knowledgecommons.popcouncil.org/departments_sbsr-pgy

Part of the Demography, Population, and Ecology Commons, Domestic and Intimate Partner Violence Commons, Family, Life Course, and Society Commons, International Public Health Commons, and the Maternal and Child Health Commons

How does access to this work benefit you? Let us know!

\section{Recommended Citation}

Adolescent Girls Empowerment Program (AGEP): Endline results-Executive summary. Lusaka:

Population Council, 2018. 
Adolescent girls in Zambia face a range of risks and vulnerabilities that challenge their healthy development from girls into young women, and they often lack the social, health, and economic assets that are necessary to mitigate these risks. The issues that confront vulnerable girls - high rates of gender-based violence, unsafe sex that puts girls at risk for unwanted pregnancies and HIV infections, school dropout, lack of economic resources and income-generating options, and lack of agency and participation are interdependent and have similar causes.

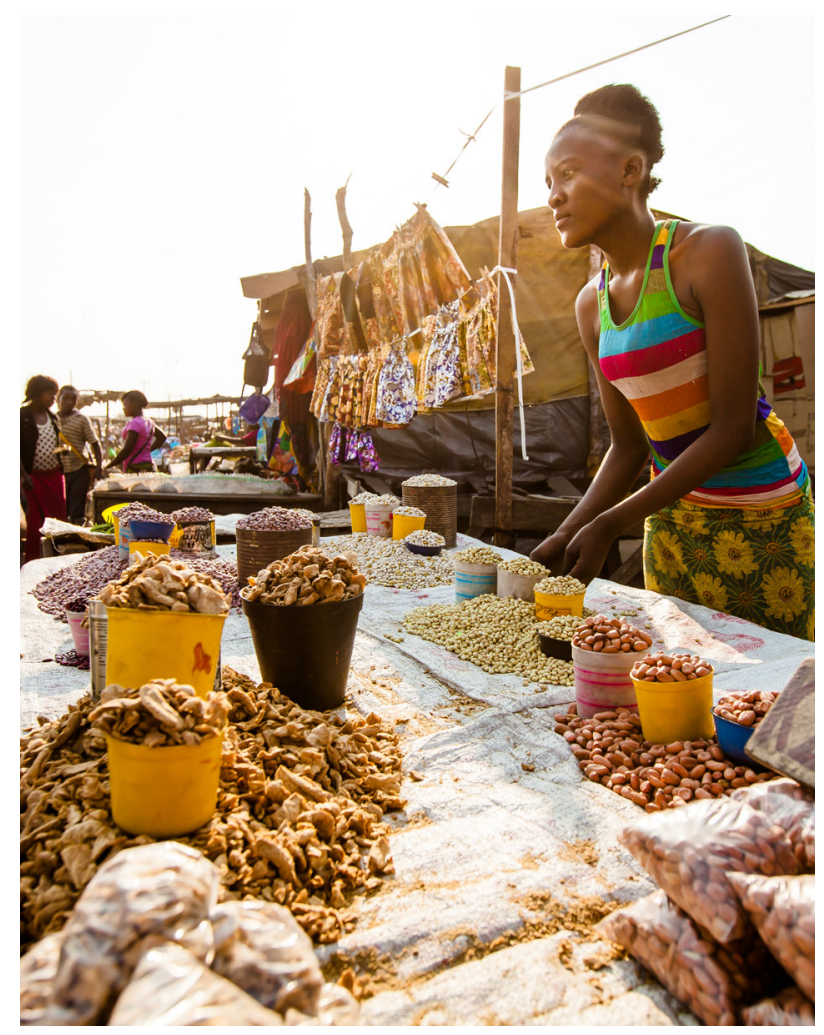

The situation is a challenging one for adolescent girls in Zambia. Across a wide range of issues-education, social support, safety, and sexual and reproductive health-girls are at a greater disadvantage compared to their male peers. The vulnerabilities confronting Zambian adolescent girls formed the basis for designing the Adolescent Girls Empowerment Programme (AGEP), which a) focused on adolescent girls aged 10-19, b) delivered community-based interventions and c) selected participants for the programme with the direct intention of capturing those girls who were the most vulnerable. AGEP was rolled out in 10 sites in four provinces in Zambia, five sites in urban areas, and five sites in rural areas, and aimed to reach 1,000 girls per site, for a total of 10,000 girls in the programme.

The theory of change behind AGEP posited that adolescent girls are empowered by building their social, health, and economic assets that they can then draw on to reduce vulnerabilities and expand opportunities. In the long term, they will then increase their likelihood of completing school, delaying sexual debut, and reducing risks of early marriages, unintended pregnancies, acquisition of HIV, and other possibly detrimental outcomes. 


\section{INTERVENTION COMPONENTS}

Safe spaces: Safe spaces were weekly girls' group meetings, implemented in partnership with YWCA Zambia, in which 20 to 30 girls met with a mentor-a young woman from their community who was hired and trained-for short training sessions on a variety of topics as well as an opportunity to discuss together their experiences in the past week. AGEP developed three curricula for adolescent girls that were used in the safe space meetings: 1) a health and life-skills curriculum, 2) a financial education curriculum, and 3) a nutrition curriculum.

Health vouchers: In partnership with the Ministry of Community Development, Mother and Child Health ( $\mathrm{MCDMCH})$, participants received a health voucher redeemable for a package of general wellness and sexual and reproductive health services at partner public and private healthcare providers. For private and NGO providers, payment was made on a "fee-for-service" basis with preapproved reimbursement rates, whereas for the public facilities, incentives for each service were paid to the District Community Health Offices (DCHO) and then distributed among the district health offices and the clinics in previously agreed-upon percentages.

Savings accounts: The Population Council worked in partnership with the National Savings and Credit Bank (Natsave) and Making Cents International to develop the Girls Dream savings accounts for AGEP girls. The Natsave accounts had very low minimum opening balances of ZMW 2.5 (US \$0.50) and any amount could be deposited or withdrawn with no fee.

\section{STUDY DESIGN}

A randomised cluster design was implemented to rigorously assess the impact of AGEP, as well as the impact of each of its core components, on the trajectories of girls' lives in their adolescent years and into early adulthood. In order to assess the effect of the safe spaces alone, as well as the added effect of the health voucher and savings account, AGEP was implemented in three versions across randomised study arms: Arm 1 included safe spaces only; Arm 2 included safe spaces and the health voucher; and Arm 3 included safe spaces, the health voucher, and the savings account. Arm 4 was a control group that received no intervention.

\section{KEY FINDINGS}

The AGEP endline evaluation focused on three main questions: 1) whether or not the changes in social, health, and economic assets (which are the mediating factors) that were observed at midline were sustained; 2) were there new impacts observed in the social, health, and economic asset indicators and 3 ) did the intervention result in any longer-term change on demographic, reproductive, and health outcomes, and if so, for which girls.

\section{AGEP IMPACT ON SOCIAL, HEALTH, AND ECONOMIC ASSETS}

Social assets: In the evaluation of AGEP, two key measures of empowerment via social assetbuilding were measured-social safety nets and self-efficacy. At midline-which measured the impact immediately at the completion of the two-year intervention-girls in the three intervention arms were more likely than girls in the control arm to have a place in the community where they could meet other girls their age apart from their home and school. However, there were no effects on self-efficacy. Two 
years post-programme, there was no longer a difference between programme and control arm girls vis-à-vis social safety nets, suggesting that the public spaces within communities that were created for girls by the programme were no longer available to them. However, with self-efficacy, an endline effect did appear. One potential explanation for this latent effect is that as girls grew older, the depth of life experiences in which they could apply the learnings and

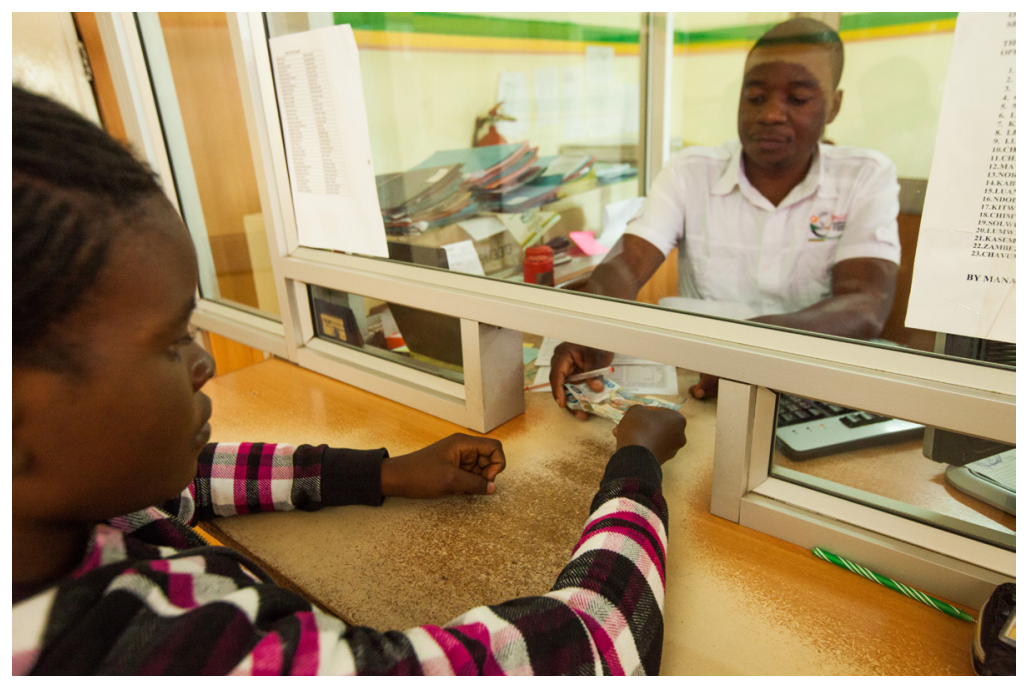
skills built from AGEP increased (especially for those with savings accounts), and therefore their beliefs in their abilities were concretised and became measurable.

Gender norms and acceptability of spousal violence: At the midline evaluation there was no effect of AGEP on either gender norms - which measured adolescent girls' attitudes on gender roles in adolescence-or acceptability of intimate partner violence (IPV) as measured by five items asking if it was acceptable for a husband to beat his wife in certain scenarios. The two-year follow-up showed that these norms and attitudes remained unchanged and there were no differences between AGEP and control arms.

Health assets: The two main health assets measured in the AGEP evaluation were sexual and reproductive health ( $\mathrm{SRH}$ ) knowledge and HIV knowledge. At midline there was an increase in SRH knowledge among girls in the programme arms relative to girls in the control, however there was no difference in the increase in HIV knowledge between the two groups. At endline, the AGEP girls continued to have higher levels of SRH knowledge compared to the control girls. However, it should be noted that overall, SRH knowledge was very low at baseline, and while it increased over the four years, it remained low at endline. Specifically, SRH knowledge approximated the equivalent of girls learning about two new methods of contraception over that period, indicating room for further education on these topics.

Economic assets: The two main economic asset indicators measured were financial literacy and savings behaviour in the past year. At midline, AGEP had a positive increase on financial literacy for all girls in the programme arms, but at endline, the overall effect on financial literacy for all programme arms no longer remained. Regarding savings behaviour, a positive effect on savings activity at midline was present and that effect remained at endline.

\section{AGEP IMPACT ON DEMOGRAPHIC, REPRODUCTIVE, AND HEALTH OUTCOMES}

Experience of violence: Overall, neither at midline nor at endline, did the AGEP intervention have an effect on girls' experience of physical or sexual violence. Important to note, however, is that experience of both kinds of violence was high at baseline, and steadily increased over the course of the study such that by ages 14-23 two-thirds of girls had experienced physical violence and by ages 15-23 half of girls had experienced sexual violence. 
Sexual behaviour: Overall, the AGEP intervention had little impact on either overall sexual activity or sexual behaviour among those who were sexually active-at both midline and endline. This included contraceptive use, condom use, and number of sexual partners. At midline, girls who were sexually active at baseline were more likely to use a condom, but by endline that effect was no longer present. This means that in the longer term, the AGEP programme did not have a positive influence on girls' safer sex behaviours. The one area of positive impact was that girls were less likely to have had transactional sex, and this effect was present at midline and at endline.

Pregnancy and marriage: Counter to the initial hypothesis, participation in AGEP did not delay the timing of pregnancies or marriages for girls in the programme arms. Overall, $39 \%$ of girls had given birth by the end of the two-year follow-up period and $28 \%$ of girls were married or living with their partner-and there was no significant difference between the programme and control arms.

Overall, in reflecting back on the theory of change, the hypothesis was that the AGEP

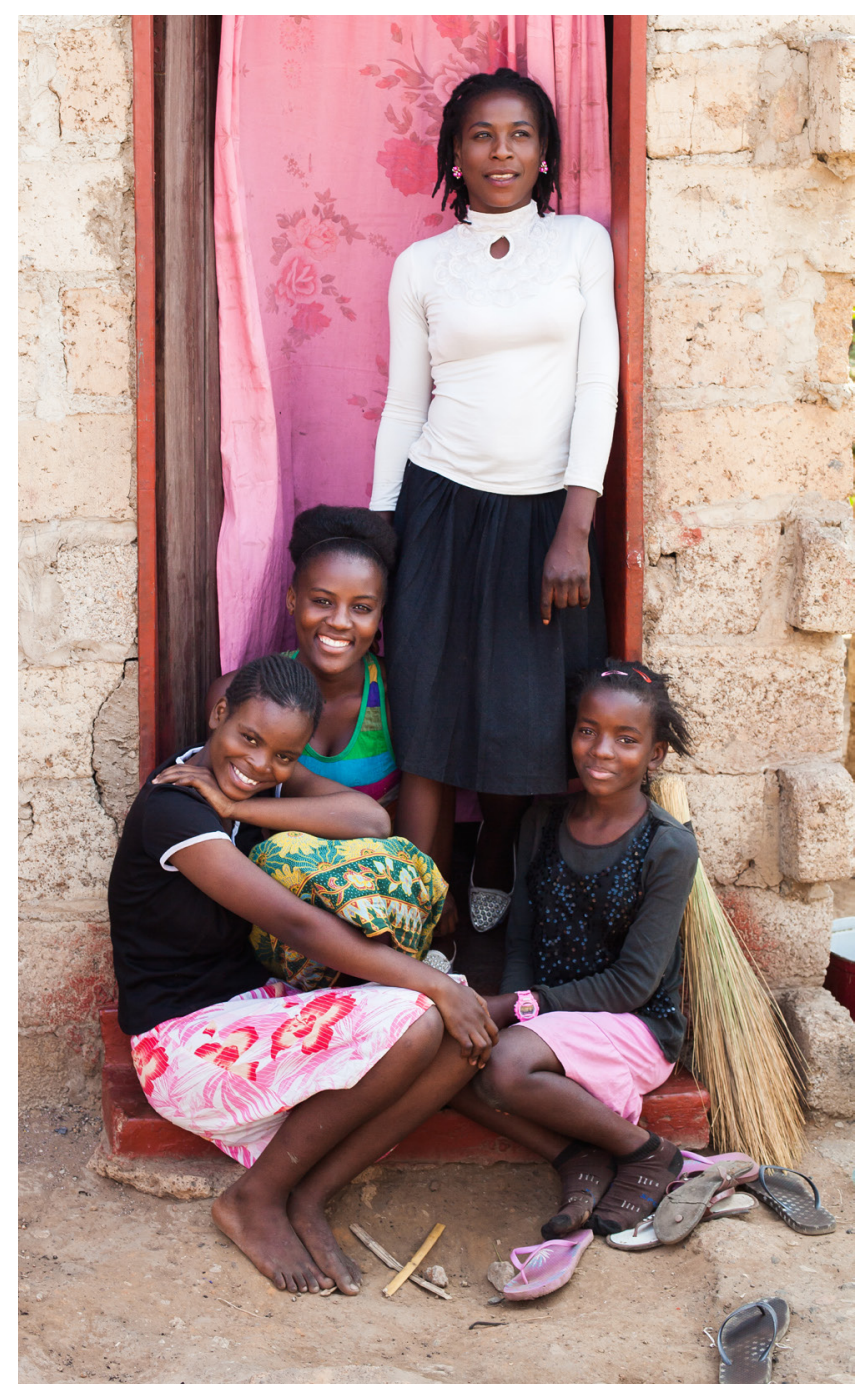
interventions would lead to increased social, health, and economic assets, and those in turn would lead to longer-term education, fertility, and health improvements. The theory of change did not prove to be confirmed in two major elements: 1) there was positive change only on a subset of the mediating factors-largely the economic assets and some of the health assets, but not on most social assets - and with modest effect sizes; and 2) the changes that did take place in the mediating outcomes were not sufficient to lead to longer-term change. Therefore, overall, the pathways to change that were anticipated did not take place. 


\section{CONCLUSIONS}

At the end of the two-year programme (the midline), the following impacts were observed:

- Improved sexual and reproductive health knowledge

- Improved access to safe spaces in the community

- Improved financial literacy

- Improved savings behaviours

- Decreased transactional sex (for girls who were sexually active at the start)

Two years after the end of the programme (the endline), the following impacts were seen:

- Improved sexual and reproductive health knowledge

- Improved self-efficacy

- Improved savings behaviours

- Decreased transactional sex (for girls who were sexually active at the start)

There were no medium or long-term effects on:

- Pregnancy/birth

- Marriage

- Sexual debut, contraceptive use, condom use, multiple sexual partners

- Equitable gender attitudes

- Experience of physical or sexual violence

Therefore, while there were some changes for the programme participants in the medium and long term, they did not translate into longer-term effects on reproductive and demographic outcomes as hypothesised via the theory of change. However, interpretation of these results is constrained by two important factors: a) a large proportion of the girls invited to the programme did not participate, and b) among those who did participate, only a sub-segment of them participated actively in the safe-spaces sessions. Knowing both who is and is not participating in a programme meant to target vulnerable adolescent girls is important-for programmatic implementation guidance as well as interpretation of results.

One of the clearest and most consistent findings was in the economic asset-building component. The savings account catalysed positive savings behaviour-both formal and informal-and had positive effects on girls' self-efficacy. This provides an indication to programmes working with adolescent girls as to the feasibility and important impact of integrating financial literacy training and access to savings opportunities into more traditional health-related programming. 


\section{RECOMMENDATIONS FOR POLICYMAKERS/IMPLEMENTERS}

1. The most vulnerable adolescent girls will likely not attend a safe spaces-only programme. While the safe spaces platform to building assets for girls can be leveraged to achieve longer-term change for girls on a range of well-being outcomes, it is likely that the most vulnerable are going to participate less, or not at all, as there are too many barriers to their participation. Programme implementers must ensure that they have the systems and budgets in place to track who is, and is not, participating. In order for programmes to be successful, they will need to include adaptations that address the needs of those sub-segments of the population (e.g., out of school, economically most disadvantaged, those living apart from their parents) to allow for enrolment and active participation.

2. Acceptability and experience of sexual and gender-based violence (SGBV) among adolescent girls and young women in Zambia is alarmingly high; it will take a more holistic, community-level programme to address this. Sixty percent of girls think that violence is acceptable in certain circumstances and over half of girls ages 14-23 have already experienced sexual violence. Given that the AGEP intervention components all worked at the level of the girls and had no impact on either of these sets of indicators, future programmes that aim to reduce SGBV among adolescents should address inequitable attitudes and norms not only with adolescent girls directly, but around addressing violence at the household, school, and community levels, as well.

3. Economic barriers will negatively influence adolescent programmes-even those that are healthoriented-and need to be addressed in adolescent programming. Household economic barriers exist, especially among the most vulnerable, that will both prevent participation, as well as prevent desired health outcomes from being achieved. Programmes should consider education support to those who are at risk of school-dropout or are out-of-school but likely to return to school. Social cash transfers may have the potential to alleviate some of the economic stress of the household, freeing up girls to participate in programmes. It may also mitigate some of the economic reasons that drive transactional sex, school dropout, and/or early marriage. Individual economic barriers, especially for older girls, also exist. Retaining an economic component to the programme, financial literacy and savings, will help address these issues. For older girls, it is likely that additional activities that promote income-generation will need to be added to programmes. 


\section{RECOMMENDATIONS FOR DONORS/RESEARCHERS}

1. More analysis can be done with the AGEP data set. There is a wealth of data available that can be used to look at the impact of the programme on additional outcomes and for various subsets of the adolescent girls cohort. The data are available at the Adolescent Data Hub. ${ }^{1}$

2. Many evidence gaps remain, and as this is a complex issue with multiple interdependent outcomes, more research is needed. More work should be done to deliver and evaluate programmes that aim to address long-term health outcomes for adolescents, that take a multi-level approach including simultaneous work with communities, households, and adolescents themselves.

3. More funding for longitudinal research on adolescents is needed. The results between midline and endline did change-including some emerging results - and therefore there is value in following cohorts of adolescents through to early adulthood to understand the effect of interventions in early and mid-adolescence on later adolescence and early adulthood. This may include effects on the transition to the workforce, quality of marital relationships, health and education outcomes for adolescents' children, etc. Additional longitudinal research, that combines data from these different levels, will help to understand the multiple factors and context that shapes adolescent transitions into adulthood

4. More research and learning from work on cash transfers are needed. More research is needed in Zambia to evaluate programmes that combine asset-building for adolescents with cash incentives at the household level. There is promising evidence coming out of Kenya2 that suggests that there may be long-term success when these are combined and implemented in early adolescence. In addition, The Transfer Project has been conducting work in Zambia,3 and other countries in sub-Saharan Africa,4 that could provide useful insights on the role of social protection cash transfer programmes. The potential of combining general cash transfers with additional programming for adolescents remains a promising area that needs more research.

The AGEP evaluation is an important contribution to the understanding of adolescent transitions and interventions in Zambia. The rigorous and long-term approach provides a level of certainty, attribution, and conclusions that are unparalleled in Zambia. It is our hope that this evaluation will contribute to the improvement of current programmes, as well as development of new programmes and funding strategies. The data show that early pregnancy, violence, and HIV are still very pressing issues for adolescents in Zambia and evidence should be used to design and implement the programmes most

${ }^{1}$ https://doi.org/10.7910/DVN/CFIUC6 
The Population Council confronts critical health and development issues-from stopping the spread of HIV to improving reproductive health and ensuring that young people lead full and productive lives. Through biomedical, social science, and public health research in 50 countries, we work with our partners to deliver solutions that lead to more effective policies, programs, and technologies that improve lives around the world. Established in 1952 and headquartered in New York, the Council is a nongovernmental, nonprofit organization governed by an international board of trustees.

Population Council, Zambia

Plot 3670, No. 4

Mwaleshi Road, Olympia Park

Lusaka, Zambia Tel: +260211295925 email: info.zambia@popcouncil.org

(C) 2018 The Population Council, Inc.

\section{For more information, please refer to the following:}

Austrian, Karen, Erica Soler-Hampejsek, Paul C. Hewett, Natalie Jackson Hachonda, and Jere R. Behrman. 2018. Adolescent Girls Empowerment Programme: Endline Technical Report. Lusaka, Zambia: Population Council.

Hewett, Paul C., Karen Austrian, Erica Soler-Hampejsek, Jere R. Behrman, Christine A. Kelly, Dela Kusi-Appouh, Fiammetta Bozzani, Barbara S. Mensch, and Minyoi Maimbolwa. 2014. Adolescent Girls Empowerment Programme: Research and Evaluation Baseline Technical Report. Lusaka, Zambia: Population Council.

Hewett, Paul C. Karen Austrian, Erica Soler-Hampejsek, Jere R. Behrman, Fiammetta Bozzani, and Natalie A. Jackson-Hachonda. 2017. Cluster randomized evaluation of Adolescent Girls Empowerment Programme (AGEP): study protocol. BMC Public Health: 17:386. DOI 10.1186/s12889-017-4280-1. 